\title{
From order to randomness: Onset and evolution of the random-singlet state in bond-disordered $\mathrm{BaCu}_{2}\left(\mathrm{Si}_{1-x} \mathbf{G e}_{x}\right)_{2} \mathrm{O}_{7}$ spin-chain compounds
}

\author{
T. Shiroka, ${ }^{1,2, *}$ F. Eggenschwiler, ${ }^{1}$ H.-R. Ott, ${ }^{1,2}$ and J. Mesot ${ }^{1,2}$ \\ ${ }^{1}$ Laboratorium für Festkörperphysik, ETH Hönggerberg, CH-8093 Zürich, Switzerland \\ ${ }^{2}$ Paul Scherrer Institut, CH-5232 Villigen PSI, Switzerland
}

(Received 24 August 2018; revised manuscript received 16 November 2018; published 8 January 2019)

\begin{abstract}
Heisenberg-type spin-chain materials have been extensively studied over the years, yet not much is known about their behavior in the presence of disorder. Starting from $\mathrm{BaCu}_{2} \mathrm{Si}_{2} \mathrm{O}_{7}$, a typical spin- $\frac{1}{2}$ chain system, we investigate a series of compounds with different extents of bond disorder, where the systematic replacement of $\mathrm{Si}$ with $\mathrm{Ge}$ results in a remodulation of the $\mathrm{Cu}^{2+}$ exchange interactions. By combining magnetometry measurements with nuclear magnetic resonance studies, we follow the evolution of the disorder-related properties from the well-ordered $\mathrm{BaCu}_{2} \mathrm{Si}_{2} \mathrm{O}_{7}$ to the maximally disordered $\mathrm{BaCu}_{2} \mathrm{SiGeO}_{7}$. Our data indicate that already a weak degree of disorder of only $5 \% \mathrm{Ge}$, apart from reducing the three-dimensional magnetic ordering temperature $T_{\mathrm{N}}$ quite effectively, induces a qualitatively different state in the paramagnetic regime. At maximum disorder our data indicate that this state may be identified with the theoretically predicted random singlet (RS) state. With decreasing disorder the extension of the RS regime at temperatures above $T_{\mathrm{N}}$ is reduced, yet its influence is clearly manifest, particularly in the features of nuclear magnetic resonance relaxation data.
\end{abstract}

DOI: 10.1103/PhysRevB.99.035116

\section{INTRODUCTION}

The study of electronic properties of physical systems in the presence of disorder spans many decades [1], starting with the strong (Anderson) localization studies in the 1950s [2] up to the present-day investigations of quantum confinement in nanostructures [3]. The breadth of phenomena taking place in disordered systems, such as quantum percolation [4], ballistic transport [5], quantum glassiness [6], or many-body localization [7], have been studied primarily theoretically, e.g., as a function of dimensionality, nature of disorder, degree of interaction, etc. Of particular interest is the physics occurring in low-dimensional quantum magnets under a varying degree of disorder. At very low temperatures and high magnetic fields, close to a quantum phase transition, disorder suppresses the global phase coherence and induces novel quantum critical behavior [8,9]. But even under less extreme conditions, the disorder-induced breaking of translational invariance promotes random couplings between individual spins and leads to a so-called random-singlet (RS) state [10-12], a regime where spins couple across arbitrary distances to form weakly bound singlets, which dominate the magnetic features and the related dynamics. What exactly happens when a regular spin-chain is exposed to an increasing degree of disorder is not well known. Until recently, progress has been slow as far as numerical simulations [13] and, especially, experimental investigations [14-16] of disordered low-dimensional systems are concerned. The main reasons include computational difficulties due to the large size of realistic disordered systems and, regarding experiments, the scarcity of suitable systems in

*Corresponding author: tshiroka@phys.ethz.ch which disorder can be easily tuned over a broad range without changing the structural character of the material.

It has been known for some time that the series of $\mathrm{BaCu}_{2}\left(\mathrm{Si}_{1-x} \mathrm{Ge}_{x}\right)_{2} \mathrm{O}_{7}$ compounds represents one of the best physical realizations of an $S=1 / 2$ Heisenberg-type spinchain system (see, e.g., Table 1 in Ref. [17]), where bond disorder can be introduced in a controlled way. For $x=$ 0 , the compound crystallizes in the orthorhombic space group Pnma $\left(D_{2 h}^{16}\right)$ with lattice constants $a=6.862 \AA, b=$ $13.178 \AA$, and $c=6.897 \AA$ [18]. Replacing Si by Ge results in isostructural compounds across the entire series (see Fig. 1), but introduces a variation in the $\mathrm{O}-\mathrm{Cu}-\mathrm{O}$ bond angle from $124^{\circ}$ in the $\mathrm{Si}$ case $(x=0)$ to $135^{\circ}$ in the Ge case $(x=1)$ [18]. As a consequence, the exchange-coupling constant almost doubles, from $J=24.1$ to $46.5 \mathrm{meV}$, when $x$ changes from 0 to 1 $[19,20]$. In this way the randomization, in the form of varying couplings, enters the magnetically relevant $\mathrm{Cu}-\mathrm{O}$ chains. The parent compound $\mathrm{BaCu}_{2} \mathrm{Si}_{2} \mathrm{O}_{7}$ orders antiferromagnetically at $T_{\mathrm{N}}=9.2 \mathrm{~K}$, i.e., at a much lower temperature than $280 \mathrm{~K}$, the equivalent of the exchange energy $J=24.1 \mathrm{meV}$. Since also the $x>0$ members have similar (or lower) $T_{\mathrm{N}}$ values, this qualifies the $\mathrm{BaCu}_{2}\left(\mathrm{Si}_{1-x} \mathrm{Ge}_{x}\right)_{2} \mathrm{O}_{7}$ series as one of the best one-dimensional (1D) systems for studying bond-disorder effects.

In previous studies, concerning the effects of randomness on the compound's magnetic properties, we focused on the extreme cases of complete order or disorder, i.e., $x=0$ and $x=0.5[21,22]$. The main result of bulk magnetization measurements and of low-temperature ${ }^{29} \mathrm{Si}$ nuclear magnetic resonance (NMR) studies, supplemented by quantum Monte Carlo calculations, was the experimental verification of the theoretically predicted random-singlet state $[10,11]$ in the $x=0.5$ case. Important ingredients of this identification are the experimental determination of the probability 


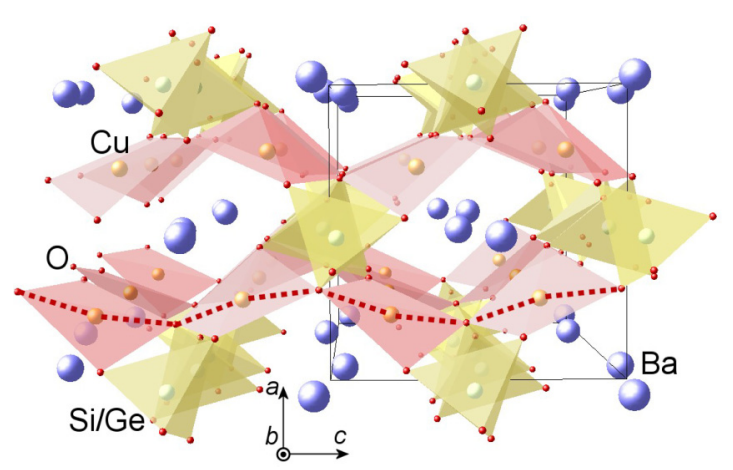

FIG. 1. Schematic crystal structure of $\mathrm{BaCu}_{2}\left(\mathrm{Si}_{1-x} \mathrm{Ge}_{x}\right)_{2} \mathrm{O}_{7}$. The corner-sharing $\mathrm{CuO}_{2}$ chains (dotted line) run along the $c$ axis. The random presence of tetrahedrally coordinated $\mathrm{Si}^{4+}$ and $\mathrm{Ge}^{4+}$ ions modulates $J$, the interaction coupling strength between $\mathrm{Cu}^{2+}$ ions.

distribution of NMR spin-lattice relaxation times $T_{1}$ and the rather unexpected (and so far unexplained) temperature dependence of $T_{1}^{-1}$ for $x=0.5$, characteristically different from that of the $x=0$ case. The current work aims to investigate the gradual development of the earlier established features, characteristic of the formation of the random-singlet state, as $x$ is varied between 0 and 0.5 . To this end we employ the same experimental techniques. In particular, we use the distinct variation of $T_{1}^{-1}(T)$ in the $0<x<0.5$ range to identify the unexpectedly rapid formation of the random-singlet state in a limited temperature interval, which grows with increasing $x$.

The paper is organized as follows: in Sec. II we present the experimental details of sample growth and characterization, as well as those related to the measurement techniques. The magnetometry and NMR data are presented and analyzed in Sec. III. In Sec. IV we discuss our main findings and compare them with those of recent muon-spin rotation studies [23]. Finally, in Sec. V, we summarize our key results.

\section{EXPERIMENTAL DETAILS}

Single crystals of $\mathrm{BaCu}_{2}\left(\mathrm{Si}_{1-x} \mathrm{Ge}_{x}\right)_{2} \mathrm{O}_{7}$ with $x=0,0.05$, $0.15,0.30,0.50$, and 0.95 were grown by the floating-zone technique, using stoichiometric amounts of reactants. Subsequently, laboratory-based x-ray diffraction (Bruker AXS D8 Discover) was used to characterize the samples, as well as to identify their orientation. Our data confirm that the considered barium copper compounds share the same orthorhombic Pnma space group [18], with only a tiny increase (2\%) in lattice parameters from the silicate $(x=0)$ to the germanate $(x=1)$ case. For all the samples, detailed structural analyses showed an excellent agreement between the nominal and the real Ge concentration $x$, as well as its homogeneous distribution within each sample.

The magnetic susceptibility was measured via standard dc magnetometry [using a superconducting quantum interference device (SQUID) by Quantum Design] at different values of the applied magnetic field $(0.01,0.1$, and $1 \mathrm{~T})$ and at temperatures between 4 and $300 \mathrm{~K}$. Since the $a$ and $c$ lattice parameters are very similar, only the $b$ axis can be easily identified. Therefore, for both the magnetometry and the NMR measurements the magnetic field was aligned along the crystalline $b$ axis.

The ${ }^{29} \mathrm{Si}$ nuclear magnetic resonance line shapes and spinlattice relaxation times $T_{1}$ were measured using standard pulsed techniques in an external field of $\mu_{0} H=7.07 \mathrm{~T}$. The NMR measurements were restricted to samples with $x \leqslant 0.5$, since at higher Ge content the ${ }^{29} \mathrm{Si}$ NMR signal progressively weakens. The field value was calibrated by means of an aluminum sample. The well established reference frequency of ${ }^{27} \mathrm{Al}$ nuclei was used to determine the absolute position of the ${ }^{29} \mathrm{Si}$ lines (typically at $59.715 \mathrm{MHz}$ ), as well as to track their shifts with varying temperature. A typical pulse duration of $\sim 5 \mu$ s was sufficient to irradiate the entire resonance, except at low temperatures in the $x=0.5$ case, where disorderbroadened NMR lines required frequency scans. For a comparison with previous results, the same NMR measurements were carried out both on aligned single crystals and on powder samples with the same chemical composition. The relatively small differences which were detected can be ascribed to the presence of crystalline anisotropies.

\section{RESULTS AND DATA ANALYSIS}

\section{A. Magnetization measurements}

The macroscopic magnetic response of samples with different Ge substitution was probed by measurements of the magnetization $M$, as a function of both temperature and field. In the first case, clear and distinct changes were noted upon $\mathrm{Ge}$ substitution $x$, as illustrated in Fig. 2, where data of the magnetic susceptibility $\chi_{x}(T)$ are displayed. All curves exhibit a broad (Bonner-Fisher-type) maximum [24], a typical feature

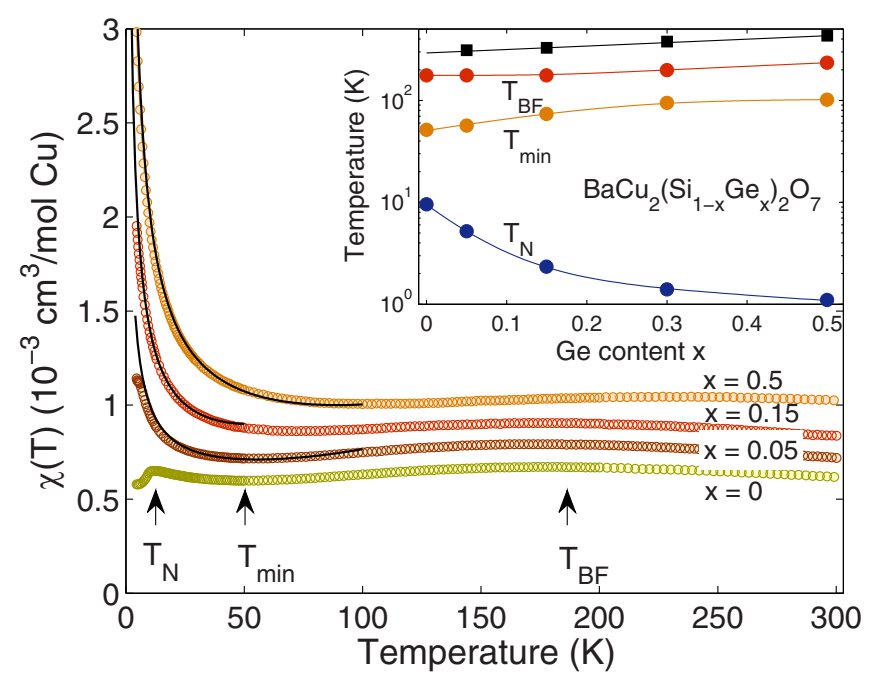

FIG. 2. Static magnetic susceptibility per mole $\mathrm{Cu}$ vs temperature at $\mu_{0} H=100 \mathrm{mT}$ for a selection of $\mathrm{BaCu}_{2}\left(\mathrm{Si}_{1-x} \mathrm{Ge}_{x}\right)_{2} \mathrm{O}_{7}$ samples with different Ge-substitution values $x$. Full lines are fits by means of Eq. (2). To improve the visibility the curves are vertically shifted by 0.2 units. Key features such as the Néel temperature, the minimum, and the Bonner-Fisher peak are marked by arrows, and their variation vs Ge content is shown in the inset together with that of the fitted $\Omega / k_{\mathrm{B}}$ values (black squares). Since the $x=0.3$ sample shows features very similar to $x=0.5$, here and elsewhere we show only the metadata for the $x=0.3$ case. 
of one-dimensional spin systems. Its position, located at $180 \mathrm{~K}$ for the $x=0$ case, shifts towards higher temperatures as the Ge content is enhanced. Since the susceptibility maximum is expected to occur at $k_{\mathrm{B}} T_{\max }=0.641 \mathrm{~J}$ and to reach a value of $\chi_{\max } \simeq 0.147 N g^{2} \mu_{\mathrm{B}}^{2} / J$ (see Eq. (30a) in Ref. [25]), a gradual increase of $T_{\max }$ (i.e., $T_{\mathrm{BF}}$ in Fig. 2) and the concomitant decrease of the corresponding susceptibility maximum clearly reflect an increase in average $J$ as the Ge content $x$ is enhanced. By using $T_{\max }=0.641 J / k_{\mathrm{B}}$, strictly valid only in the uniform- $J$ case, we obtain $J / k_{\mathrm{B}}=280.8 \mathrm{~K}$, in excellent agreement with previously derived values from magnetometry [18] or neutron diffraction studies $(J=24.1 \mathrm{meV}$, equivalent to $280 \mathrm{~K}$ ) [19]. For $x>0$, the same approach can still provide an average $J$ value, yet it cannot fully capture the true nature of the disorder-induced effects (see below).

The sharp maximum in $\chi(T)$ at low temperatures indicates the transition to a 3D antiferromagnetic state, ${ }^{1}$ with the value of $T_{\mathrm{N}}$ reflecting the intensity of the residual intrachain interactions. The position of the $T_{\mathrm{N}}$ peak shifts towards lower temperatures as the Ge substitution is enhanced (see also Ref. [18]). The temperature dependence of $T_{\mathrm{N}}, T_{\mathrm{BF}}$, as well as that of an intermediate minimum are shown in the inset of Fig. 2.

Measurements of the magnetization $M(H)$ as a function of field, from $\mu_{0} H=0$ up to $5 \mathrm{~T}$, were also performed at selected temperatures. A change in applied field by two orders of magnitude did not show essential variations in susceptibility, except for a better signal-to-noise ratio at higher fields. The $M(H)$ data (not shown here) reveal a linear variation of $M$ vs $H$, with an increasing slope as the temperature decreases. The observed linearity indicates the low effective magnetic moment of $\mathrm{Cu}^{2+}$ ions, a typical feature of the quantum magnetism of spin chains.

Fitting the susceptibility data $\chi(T)$ is not straightforward, since analytical formulas exist only in the limiting cases of a uniform $J(x=0)$, or of an alternating-exchange $J_{1}-J_{2}$ $(x=0.5)$ Heisenberg spin-chain model. In earlier work, magnetometry data of quasi-1D spin chain systems were analyzed by using the expression $[18,25]$

$$
\chi(T)=\chi_{0}+\chi_{\mathrm{CW}}(T)+\chi_{\mathrm{spin}}(T) .
$$

Here $\chi_{0}$ is a temperature-independent term due to core diamagnetism and Van Vleck paramagnetism, $\chi_{\mathrm{CW}}=C /(T-\Theta)$ is a Curie-Weiss contribution, and $\chi_{\text {spin }}(T)$ represents the susceptibility of the 1D spin system. The Curie-type term was introduced to account for the low-temperature upturn, typically being attributed to impurities, defects, or intrinsic weak ferromagnetism.

Although formal fits using Eq. (1) follow fairly well the experimental data, this approach has some serious drawbacks. First, it fails to capture the physical meaning of the low- $T$ divergence, which in our case is not due to impurities, but rather reflects the low-energy states associated with the weakly bound singlets. In the theoretically predicted

\footnotetext{
${ }^{1}$ While at low $x$ values $T_{\mathrm{N}}$ is clearly visible from the $\chi(T)$ data, at higher $x$ values, due to the rapid divergence of $\chi(T)$, it appears only as a small change in slope.
}

random-singlet state, their magnetic response is modeled by [11]

$$
\chi(T) \sim T^{-1} \ln ^{-2}(\Omega / T),
$$

with $\Omega$ a cutoff energy. In previous work, we showed that for $x=0.5$, Eq. (2) describes very well the upturn in $\chi(T)$, with a reasonable value of the fit parameter $\Omega$ [21]. Second, Eq. (1) provides only an average, $x$-dependent exchange coupling value $J$ which, in Ref. [21], was clearly shown to be insufficient to capture the complexity of spin chains with bond randomness.

As demonstrated in Fig. 2, by using Eq. (2) we obtain reasonably good fits for all the $x>0$ susceptibility curves. Obviously, to capture the influence of randomness, we had to restrict the fit range from ca. $1.5 T_{\mathrm{N}}$ to $0.5 T_{\mathrm{BF}}$. This choice is justified because it avoids the complications of the $3 \mathrm{D}$ ordering effects at very low temperatures, while still exploring the quantum effects of the random-singlet state, which lose dominance at higher temperatures. The inset of Fig. 2 shows the variation of $\Omega$ with $x$ (black squares).

\section{B. Magnetic resonance measurements}

To gain further insight into the low-temperature spin dynamics and the local magnetic susceptibility, we performed detailed NMR measurements. Since the line shift $K$ reflects the intrinsic spin susceptibility at zero frequency $\chi(q=$ $0, \omega=0)$, while the spin-lattice relaxation rate tracks the (low-energy) spin fluctuations, NMR is among the best techniques for studying the static and dynamic properties of lowdimensional spin systems (see, e.g., Ref. [26]).

a. NMR linewidths and shifts. The evolution of the ${ }^{31} \mathrm{P}$ NMR line shapes with temperature for an $x=0.15$ single crystal is shown in Fig. 3. The linewidth is approximately constant at high temperatures, but exhibits a divergent increase starting at an onset of $\sim 80 \mathrm{~K}$. Similar results were obtained also for other concentrations $x$, but the onset temperature increases as the Ge substitution level $x$ is enhanced. An NMR linewidth, rapidly varying with decreasing temperature, directly reflects the growing distribution of the local magnetic susceptibilities and is in good agreement with theoretical predictions for random Heisenberg chains [11]. In general, an increase in Ge content causes a growth of disorder, reflected in enhanced linewidths already at room temperature. This is clearly shown in the lower inset of Fig. 3 where, at $T>$ $100 \mathrm{~K}$ (dominated by classical disorder effects), the FWHM linewidths in the $x=0.15$ case are a factor of 2 larger than for $x=0.05$. A further increase in disorder (for $x=0.50$ ) does not imply an additional increase in width at $T>100 \mathrm{~K}$, but rather a FWHM saturation. This is to be contrasted with the faster decrease of the stretching coefficient $\beta$ with decreasing temperature at higher $x$ values (see below).

While linewidths exhibit a significant increase at low $T$, line shifts $K(T)$ are generally quite small. Even in case of single-crystalline samples, the total ${ }^{29} \mathrm{Si}$ NMR line shifts are less than $0.03 \%$, which is not surprising considering the $1 \mathrm{D}$ nature of $\mathrm{BaCu}_{2}\left(\mathrm{Si}_{1-x} \mathrm{Ge}_{x}\right)_{2} \mathrm{O}_{7}$. At the same time, typical of 1D materials, the wide distribution of local Knight shifts of magnetic origin implies a strong increase of linewidths at low temperature. As shown in Fig. 3, $K(T)$ represents only a tiny 


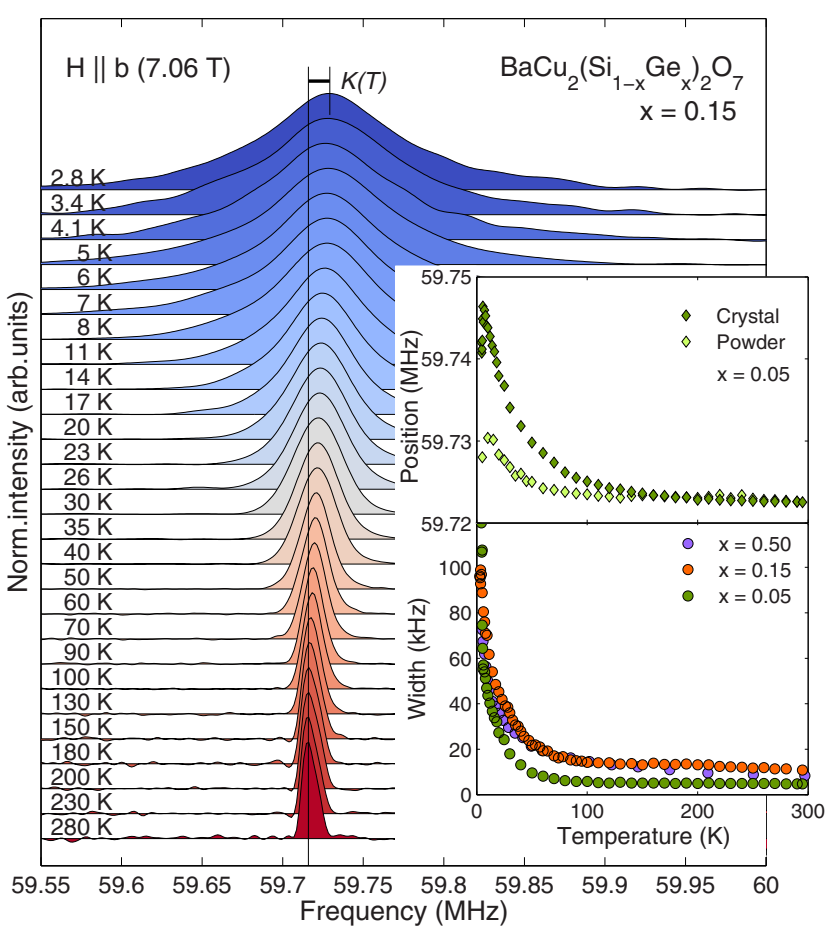

FIG. 3. Evolution of ${ }^{29} \mathrm{Si}$ NMR lines with temperature in $\mathrm{BaCu}_{2}\left(\mathrm{Si}_{1-x} \mathrm{Ge}_{x}\right)_{2} \mathrm{O}_{7}$ for an $x=0.15$, single-crystal sample with the $b$ axis aligned along the field. The upper inset shows typical line shifts for the $x=0.05$ case, where both powder and single-crystal data exhibit a cusp at $T_{\mathrm{N}}$ (other samples show similar features). The lower inset highlights the low-temperature increase of linewidth for different $x$ values. For $T>100 \mathrm{~K}$, the wider lines in the $x=0.15$ and 0.50 cases indicate a disorder-induced broadening.

fraction of the linewidth, thus making it difficult to precisely evaluate its value. Line shape measurements on powder samples show a similar behavior (upper inset in Fig. 3), with small differences most likely ascribed to the presence of crystalline anisotropies. For all $x>0$ cases, $K(T)$ shows first a progressive increase with decreasing temperature, then it reaches a sharp cusp-like maximum at the respective ordering temperature $T_{\mathrm{N}}$. These results confirm (at a microscopic level) the magnetization data shown in Fig. 2 and are consistent with predictions for spin chains in local transverse staggered fields, as reported in previous work on single crystals of $\mathrm{BaCu}_{2} \mathrm{Si}_{2} \mathrm{O}_{7}$ $(x=0)$ [27]. It is worthwhile noting that, unlike the $x=0$ case, where below $T_{\mathrm{N}}$ the two components of the NMR line can easily be resolved (see Fig. 2 in Ref. [27]), for $x>0$, due to disorder-induced broadening, this is not possible. In this case, we have direct access only to the total shift, i.e., to the average shift of the two components, whose position is relatively insensitive to disorder. Indeed, the cusp-like maximum in $K(T)$ observed for $x>0$ reproduces perfectly the average-shift results reported for the $x=0$ case [27].

b. NMR relaxation rates. In spin-chain compounds, the NMR spin-lattice relaxation rate $1 / T_{1}$ has been shown to capture efficiently the subtle changes in spin fluctuations as a function of temperature [21]. Below we demonstrate that this applies also to the case of a varying degree of disorder.

The nuclear spin relaxation is mainly driven by the electronic spin fluctuations on $\mathrm{Cu}^{2+}$ ions, coupled to ${ }^{29} \mathrm{Si}$ nuclei via the $q$-dependent hyperfine interactions $A(\boldsymbol{q})$ :

$$
\frac{1}{T_{1 z}} \propto \hbar^{2} k_{\mathrm{B}} T \gamma_{n}^{2} \sum_{\substack{\alpha=x, y, z \\ \boldsymbol{q}}}\left[\tilde{A}_{x \alpha}^{2}(\boldsymbol{q})+\tilde{A}_{y \alpha}^{2}(\boldsymbol{q})\right] \frac{\chi_{\alpha \alpha}^{\prime \prime c}\left(\boldsymbol{q}, \omega_{n}\right)}{\omega_{n}} .
$$

Here $\omega_{n}$ is the resonance frequency, the $q$-dependent factor $A(\boldsymbol{q})$ is the NMR geometric form factor, while the $\omega$ dependent term $T \chi_{\alpha \alpha}^{\prime \prime c}\left(\boldsymbol{q}, \omega_{n}\right) / \omega_{n} \propto S_{\perp}(\boldsymbol{q}, \omega)$ is the dynamic structure factor. In general, the nuclear relaxation rate involves both isotropic and anisotropic components of either hyperfine or dipolar origin [28]. As we discuss in Sec. IV, the latter, which mediate both transverse and longitudinal spin fluctuations, are not relevant in our case and do not appear in Eq. (3), the standard expression. Thus, the longitudinal relaxation depends only on the transverse component of the fluctuating fields [29].

Figure 4(a) shows the magnetization recovery curves $M(t)$ at different temperatures for the $x=0.15$ case, with the crystalline $b$ axis oriented along the field. At a first approximation, the curves were fitted using $M(t)=1-\exp \left(-t / T_{1}\right)^{\beta}$, with $\beta$ a stretching exponent varying between 0 and 1 . Clearly a lowering of temperature induces not only a shift of the curves towards longer delays but, most importantly, it implies an enhanced stretching (i.e., a reduction of $\beta$ ).

An overview of the $1 / T_{1}$ relaxation rates for different $x$ values is shown in Fig. 4(b), the most important result in this work. Apart from the low-temperature ordered-phase region, the spin-lattice relaxation rate increases with temperature for all $x$ values. Its exact behavior depends on the degree of disorder, however. Thus, in the disorder free $x=0$ case, $1 / T_{1}(T)$ follows roughly a quadratic $T$ dependence. However, in the presence of disorder $(x>0), 1 / T_{1}(T)$ increasingly tends to a linear-in- $T$ variation, which becomes close to perfect in the maximum-disorder $x=0.5$ case. In spite of recent attempts $[13,30]$, the currently available theoretical models cannot successfully reproduce the experimental relaxation data for $x>$ 0 , i.e., taking into account the random-singlet state. As for the magnetically ordered phase, the low-temperature results for various $x$ values are shown in the inset of Fig. 4(b) $(x=0.5$ is not included, since no ordering is observed above $1.5 \mathrm{~K}$ ). The sharp peaks observed at the relevant $T_{\mathrm{N}}$ values reflect the critical slowing down of fluctuations near a second-order phase transition [31,32], which signal the antiferromagnetic transition. Clearly, the decrease of $T_{\mathrm{N}}$ with $x$, evident in the $1 / T_{1}(T)$ data, fully confirms the magnetization results (see inset in Fig. 2).

The consequences of the random-singlet (RS) state are most clearly visible in Fig. 4(c), where we report the evolution of the stretching exponent $\beta$ with temperature for different $x$ values. While for $x=0$ the magnetization recovery curves are always well reproduced by single-exponential $(\beta=1)$ fits (see Fig. 3(b) in Ref. [21]), for $x=0.05, \beta$ is approximately 1 only down to $\sim 70 \mathrm{~K}$. As $x$ increases, $\beta$ starts to depart distinctly from 1 at increasingly higher temperatures. This is confirmed by phenomenological $\beta(T)=1-a \exp \left(-T / T_{\mathrm{RS}}\right)$ fits, which indicate higher $T_{\mathrm{RS}}$ values, i.e., the persistence of the RS regime over a wider $T$ range, as $x$ increases. In previous work, we have shown that a stretched exponential 

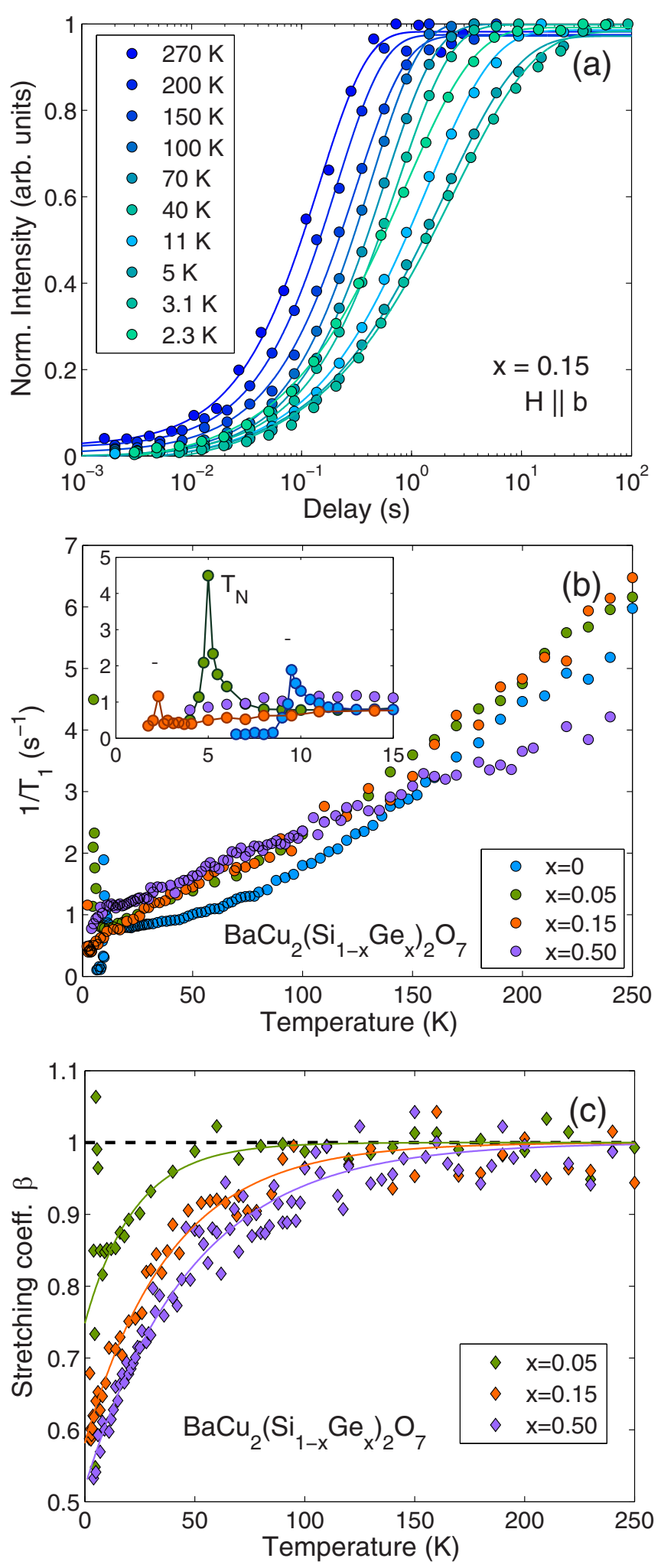

FIG. 4. (a) Raw relaxation data and relevant fits for an $x=0.15$, $b$-axis oriented $\mathrm{BaCu}_{2}\left(\mathrm{Si}_{1-x} \mathrm{Ge}_{x}\right)_{2} \mathrm{O}_{7}$ single crystal. The multidecade recovery of magnetization at low temperatures reflects the significant decrease of the stretching coefficient $\beta$. (b) Inverse spin-lattice relaxation time vs temperature for a selection of $\mathrm{BaCu}_{2}\left(\mathrm{Si}_{1-x} \mathrm{Ge}_{x}\right)_{2} \mathrm{O}_{7}$ samples with different Ge-substitution values $x$. Inset: relaxation rates at low temperature. Peaks denote magnetic-transition values $T_{\mathrm{N}}$, arising from 3D interchain interactions, which decrease with relaxation, corresponds to a distribution of relaxation rates arising from a multitude of different local environments $[21,27]$. In our case, this corresponds to coexisting electronspin singlets with different coupling strengths, i.e., to the RS scenario. The earlier departure of $\beta$ from 1 at higher $x$ values confirms that the RS regime emerges more easily in such cases.

\section{DISCUSSION}

The NMR linewidths vs temperature reported in Fig. 3 exhibit a very broad frequency/field distribution for all the $x>0$ cases, remarkably different from the pure $x=0$ case, where the linewidths are almost constant with temperature [27]. If the broadening were due to disorder effects only, at low $x$ values the ${ }^{29} \mathrm{Si}$ nuclei would still probe (on average) practically the same environment as in the disorder-free case. However, the unusually large linewidths we observe already for $x=0.05$ rule out the hypothesis of a disorder-induced low-temperature broadening. Instead, they strongly suggest that it is the $\mathrm{RS}$ pairing in the individual $\mathrm{Cu}^{2+}$ chains to cause an extremely varied magnetic-field distribution (echoing the distribution of the inverse exchange constants), which is then reflected in the NMR linewidths.

To understand the observed NMR relaxation rates, we recall that the typical 1D spin-chain $(\omega, \boldsymbol{q})$ dispersion, measured in inelastic neutron scattering, covers an area limited by a single sine curve from above and a double sine from below, the so-called two-spinon continuum [33-36]. Due to the low radiofrequency energies employed in NMR (some $\mu \mathrm{eV}$ ), the latter explores only the low-energy $1 \mathrm{D}$ spin excitations. Hence only the $q \approx 0$ and $q \approx \pi$ parts of the dynamical susceptibility $\chi^{\prime \prime}(T)$ contribute to the NMR relaxation. In the Luttinger liquid (LL) theory, $\chi^{\prime \prime}(T, q \approx 0)$ is temperature-independent [37] implying that $1 / T_{1}(T) \sim T$ [38], a result practically unaffected by form factor enhancements. At nonzero temperatures, nonlinear terms beyond the LL model imply a more complex behavior, $1 / T_{1}(T) \sim T \sqrt{T \ln ^{2}\left(J / k_{B} T\right) / \omega_{n}}$ [39]. However, since in our case the expected $1 / \sqrt{H}$ dependence of $1 / T_{1}$ was not observed experimentally, a model explaining also our observations is still missing. As for $\chi^{\prime \prime}(T, q \approx \pi)$, this is divergent at zero energies for $T \rightarrow 0$, as expected for a system with quasi-long-range AFM order, and its $\boldsymbol{q}$-integrated expression without the form factor would give $1 / T_{1}(T) \sim$ $T^{(1 / 2 \alpha)-1}$, where $\alpha=1 / 2$ in zero field [38]. Since at low temperature the spectral weight is concentrated around the AFM point of the Brillouin zone, for $q \approx \pi$ one has to include also the NMR form factor, which finally gives a power-law dependence for $1 / T_{1}(T)$ [see $x=0$ data in Fig. 4(b)].

While the above considerations are strictly valid only for $x=0$, intermediate $x>0$ cases are characterized by an increasing loss of translational invariance due to disorder. The translational inequivalence of sites introduces a multitude of

increasing $x$. (c) Stretching coefficient $\beta$ vs temperature. Smooth lines are phenomenological fits. The horizontal dashed line indicates the $\beta=1$ value expected in case of lack of disorder. Low- $T$ anomalies reflect the influence of the magnetic order with onset at $T_{\mathrm{N}}$. 
local nuclear relaxation times, notably different from the case of a chain with an average $J_{\text {eff }}=\left(J_{\mathrm{Si}}+J_{\mathrm{Ge}}\right) / 2 \approx 37 \mathrm{meV}$ value. This makes it difficult to account theoretically for the observed almost-linear $1 / T_{1}(T)$ behavior. Indeed, the reported data are not described properly by existing theories $[13,30]$, which even predict a reduction of $1 / T_{1}(T)$ with increasing temperature.

We recall that there are two key problems in comparing experimental data with theoretical predictions: first, theory refers typically to nuclei belonging to the same ion $\left(\mathrm{Cu}^{2+}\right.$ in this case) as the electronic spins, while our experiment uses ${ }^{29} \mathrm{Si}$ as a probe (the copper NMR signal relaxes too fast). Second, Ge sites have to be removed from the average and the effects of a nonzero magnetic field on low- $J$ bonds (increasingly important at low temperatures) have to be considered. In addition, the obtained $T_{1}$ values contain not only the transverse spin fluctuations (which can be calculated), but also a contribution from anisotropic dipolar couplings $A_{\text {dip }}(q)$, which mediate transverse and longitudinal spin fluctuations [28,29]. However, this is negligible in our field range, or can be minimized by a suitable orientation of the single crystal [40]. The above complexities make it clear why a reliable theory of $1 / T_{1}(T)$ relaxation for $1 \mathrm{D}$ spin-chains with disorder is still not available. Nevertheless, by considering the experimental data and the translational invariance breaking for $x>0$, we can envisage a shift in spectral weight as the main cause for the disorder-induced modification of $1 / T_{1}(T)$.

The $x=0$ compound can sustain correlated antiferromagnetic fluctuations, whose main weight is at $q \approx \pi$. As shown in Ref. [21], the experimental ${ }^{29} \mathrm{Si} 1 / T_{1}(T)$ data can only be fitted by including the NMR form factor, which results in a power law, $1 / T_{1} \sim T^{\alpha}$ with $\alpha>1$. A convincing presentation of the differing influences of $q=0$ and $q=\pi$ excitations and of the site of the probing nuclei is given, e.g., in Ref. [41]. This representation, however, becomes "blurred" at $x>0$, implying a weight transfer towards $q \approx 0$, in turn reflected in a more linear $1 / T_{1}$ behavior, as is indeed observed.

Since there is no agreed upon measure of randomness, we choose to represent our key results in Fig. 5 as a function of the Ge concentration $x$. Besides the ordering temperature $T_{\mathrm{N}}$, derived from the peak in both the NMR shift and the relaxation data, we report here also the crossover temperature $T_{\mathrm{RS}}$. The latter corresponds to the phenomenological temperature parameter appearing in $\beta(T)=1-a \exp \left(-T / T_{\mathrm{RS}}\right)$, used to describe the stretching exponential data shown in Fig 4(c). In view of its exponential $T$ dependence, $\beta\left(3 T_{\mathrm{RS}}\right)=0.97 \approx 1$. Hence the influence of quantum effects due to randomness may be regarded as negligible at this temperature. The fast increase of $T_{\mathrm{RS}}$ from $x=0$ to 0.05 , followed by a saturation above $x=0.15$, clearly indicates that the RS regime sets in already at a small degree of disorder, and it then persists up to higher $x$ values. Such behavior is confirmed also by the raw relaxation-rate data in Fig. 4(b), where all the $1 / T_{1}(T)$ curves for $x>0$ show a qualitatively similar behavior, distinctly different from $x=0$. The above results can be understood by considering that the average length of the chain segments with constant coupling $J_{\mathrm{Si}}$ becomes shorter as $x$ increases. At a macroscopic scale, also the magnetization curves show how a relatively small increase of disorder can produce significant

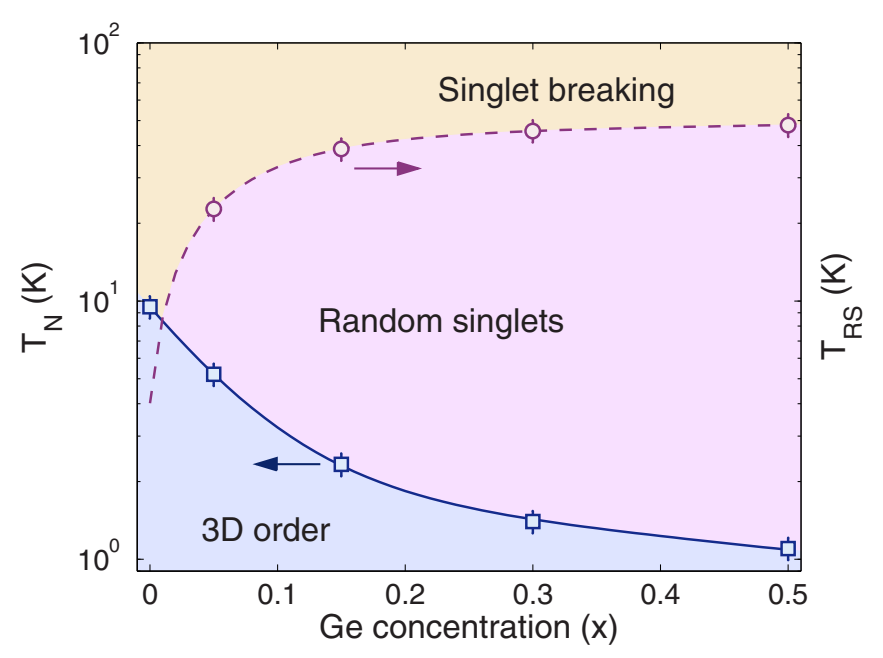

FIG. 5. Ordering temperature $T_{\mathrm{N}}$ and RS-regime crossover temperature $T_{\mathrm{RS}}$ (see text) vs Ge concentration $x . T_{\mathrm{RS}}$ reaches its asymptotic value already for $x>0.1$, suggesting that even a weak degree of disorder is sufficient to achieve the random-singlet state. Schematically, the RS regime exists between the lower 3D magnetic order phase boundary and the upper thermal singlet-breaking region. $T_{\mathrm{N}}$ data for $x=0.5$ is from Ref. [23]. Lines are guides to the eye, with the dashed line representing a crossover.

changes in the physical properties. This is particularly evident in the low-temperature regime, where $\chi(T)$ of the $x=0$ sample tends to a finite value, whereas those of the other compounds tend to diverge.

Finally, we recall that earlier muon-spin rotation $(\mu \mathrm{SR})$ and specific-heat measurements, focusing on features of the ordered magnetic phase of $\mathrm{BaCu}_{2}\left(\mathrm{Si}_{1-x} \mathrm{Ge}_{x}\right)_{2} \mathrm{O}_{7}$, came to similar conclusions [23]. These studies found that, even in case of weak disorder, the magnetically ordered state established below $T_{\mathrm{N}}$ is rather inhomogeneous. In addition, since in the presence of disorder the concept of quasimomentum and the related spin-wave excitation picture break down, the low-temperature $\left(T \ll T_{\mathrm{N}}\right) x$-dependent specific-heat data, $C(T) \propto T^{\alpha}$, exhibit a strong decrease of $\alpha$ with increasing $x$ (at fixed temperature). The latter is a clear indication of an increasing density of low-energy excitations with $x$. These findings are compatible with our magnetization and NMR results, indicating for all $x>0$ the formation of an RS state above the respective ordering temperatures $T_{\mathrm{N}}$.

\section{SUMMARY}

By using magnetometry and nuclear magnetic resonance measurements we investigated the random Heisenberg spinchain system $\mathrm{BaCu}_{2}\left(\mathrm{Si}_{1-x} \mathrm{Ge}_{x}\right)_{2} \mathrm{O}_{7}$. Since a substitution of $\mathrm{Si}$ for $\mathrm{Ge}$ modulates the exchange couplings $J$ between $\mathrm{Cu}^{2+}$ ions, by systematically varying the Ge content $x$, we could explore the transition from the purely ordered $x=0$ to the fully random $x=0.5$ case.

The random-singlet (RS) regime, already demonstrated to occur at maximum disorder in the $\mathrm{BaCu}_{2} \mathrm{SiGeO}_{7}$ case [21], was shown to emerge also for intermediate $x$ values. Thus, for $x>0$, magnetic susceptibility curves show a low-temperature divergence, perfectly fitted by a random-singlet model with 
increasingly higher cutoff energy. Similarly, both ${ }^{29} \mathrm{Si}$ NMR relaxation rates $1 / T_{1}(T)$ and the stretching coefficients $\beta(T)$ show clear indications of an RS scenario. These include a change in functional form and a departure from 1, respectively, both of which are compatible with the development of a multitude of spin-singlets with different coupling strengths. In all cases, the RS regime was shown to occur slightly above a (possible) 3D magnetic-ordering temperature and to cover increasingly wider temperature ranges as $x$ increases (up to ca. $120 \mathrm{~K}$ for $x=0.5$ ). Surprisingly, since the RS state sets in already in the $x=0.05$ case, we conclude that even a small degree of disorder is sufficient to induce it, albeit within a rather restricted temperature range.

The experimental evidence reported here, of the occurrence of an RS regime in spin-chains with a varying degree of disorder, is still missing a proper theoretical framework. Since to date only the fully random $x=0.5$ case has been addressed, future theoretical efforts should consider also the more subtle cases of intermediate disorder.

\section{ACKNOWLEDGMENTS}

The authors thank A. Feiguin (Northeastern University, Boston) and Ch. Rüegg (PSI) for useful discussions and A. Zheludev (ETH) for remarks on the first draft. The $\mathrm{BaCu}_{2} \mathrm{SiGeO}_{7}$ samples used in this work were prepared in the early 2000s in the group of Prof. K. Uchinokura at the University of Tokyo. This work was supported in part by the Schweizerische Nationalfonds zur Förderung der Wissenschaftlichen Forschung (SNF) through Grant No. 200021169455.
[1] 50 Years of Anderson Localization, edited by E. Abrahams (World Scientific, Singapore, 2010).

[2] P. W. Anderson, Absence of diffusion in certain random lattices, Phys. Rev. 109, 1492 (1958).

[3] H. Oka, O. O. Brovko, M. Corbetta, V. S. Stepanyuk, D. Sander, and J. Kirschner, Spin-polarized quantum confinement in nanostructures: Scanning tunneling microscopy, Rev. Mod. Phys. 86, 1127 (2014).

[4] G. Schubert and H. Fehske, Quantum percolation in disordered structures, in Quantum and Semi-classical Percolation and Breakdown in Disordered Solids, edited by B. K. Chakrabarti, K. K. Bardhan, and A. K. Sen, Lecture Notes in Physics, (Springer-Verlag, Berlin, 2009), Vol. 762, Chap. 5, pp. 135162.

[5] K. W. H. Stevens, One-dimensional ballistic transport of electrons, J. Phys. C: Solid State Phys. 20, 5791 (1987).

[6] C. Chamon, Quantum Glassiness in Strongly Correlated Clean Systems: An Example of Topological Overprotection, Phys. Rev. Lett. 94, 040402 (2005).

[7] F. Alet and N. Laflorencie, Many-body localization: An introduction and selected topics, C. R. Phys. 19, 498 (2018).

[8] A. Zheludev and T. Roscilde, Dirty-boson physics with magnetic insulators, C. R. Phys. 14, 740 (2013).

[9] V. Zapf, M. Jaime, and C. D. Batista, Bose-Einstein condensation in quantum magnets, Rev. Mod. Phys. 86, 563 (2014).

[10] C. Dasgupta and S.-K. Ma, Low-temperature properties of the random Heisenberg antiferromagnetic chain, Phys. Rev. B 22, 1305 (1980).

[11] D. S. Fisher, Random antiferromagnetic quantum spin chains, Phys. Rev. B 50, 3799 (1994).

[12] O. Motrunich, S.-C. Mau, D. A. Huse, and D. S. Fisher, Infiniterandomness quantum Ising critical fixed points, Phys. Rev. B 61, 1160 (2000).

[13] Y.-R. Shu, M. Dupont, D.-X. Yao, S. Capponi, and A. W. Sandvik, Dynamical properties of the $S=1 / 2$ random Heisenberg chain, Phys. Rev. B 97, 104424 (2018).

[14] F. Hammerath, S. Nishimoto, H.-J. Grafe, A. U. B. Wolter, V. Kataev, P. Ribeiro, C. Hess, S.-L. Drechsler, and B. Büchner, Spin Gap in the Zigzag Spin-1/2 Chain Cuprate $\mathrm{Sr}_{0.9} \mathrm{Ca}_{0.1} \mathrm{CuO}_{2}$, Phys. Rev. Lett. 107, 017203 (2011).
[15] N. Hlubek, P. Ribeiro, R. Saint-Martin, A. Revcolevschi, G. Roth, G. Behr, B. Büchner, and C. Hess, Ballistic heat transport of quantum spin excitations as seen in $\mathrm{SrCuO}_{2}$, Phys. Rev. B 81, 020405 (2010).

[16] Y. Utz, F. Hammerath, R. Kraus, T. Ritschel, J. Geck, L. Hozoi, J. van den Brink, A. Mohan, C. Hess, K. Karmakar, S. Singh, D. Bounoua, R. Saint-Martin, L. Pinsard-Gaudart, A. Revcolevschi, B. Büchner, and H.-J. Grafe, Effect of different in-chain impurities on the magnetic properties of the spin chain compound $\mathrm{SrCuO}_{2}$ probed by NMR, Phys. Rev. B 96, 115135 (2017).

[17] C. Broholm et al., Magnetized states of quantum spin chains, in High Magnetic Fields, edited by C. Berthier, L. P. Lévy, and G. Martinez, Lecture Notes in Physics (Springer-Verlag, Berlin, 2002), Vol. 595, Chap. 8, pp. 211-234.

[18] T. Yamada, Z. Hiroi, and M. Takano, Spin-1/2 quantum antiferromagnetic chains with tunable superexchange interactions found in $\mathrm{BaCu}_{2}\left(\mathrm{Si}_{1-x} \mathrm{Ge}_{x}\right)_{2} \mathrm{O}_{7}$, J. Solid State Chem. 156, 101 (2001).

[19] I. Tsukada, Y. Sasago, K. Uchinokura, A. Zheludev, S. Maslov, G. Shirane, K. Kakurai, and E. Ressouche, $\mathrm{BaCu}_{2} \mathrm{Si}_{2} \mathrm{O}_{7}$ : A quasi-one-dimensional $S=1 / 2$ antiferromagnetic chain system, Phys. Rev. B 60, 6601 (1999).

[20] M. Kenzelmann, A. Zheludev, S. Raymond, E. Ressouche, T. Masuda, P. Böni, K. Kakurai, I. Tsukada, K. Uchinokura, and R. Coldea, Spin waves and magnetic ordering in the quasi-onedimensional $S=1 / 2$ antiferromagnet $\mathrm{BaCu}_{2} \mathrm{Si}_{2} \mathrm{O}_{7}$, Phys. Rev. B 64, 054422 (2001).

[21] T. Shiroka, F. Casola, V. Glazkov, A. Zheludev, K. Prša, H.-R. Ott, and J. Mesot, Distribution of NMR Relaxations in a Random Heisenberg Chain, Phys. Rev. Lett. 106, 137202 (2011).

[22] T. Shiroka, F. Casola, W. Lorenz, K. Prša, A. Zheludev, H.-R. Ott, and J. Mesot, Impact of strong disorder on the static magnetic properties of the spin-chain compound $\mathrm{BaCu}_{2} \mathrm{SiGeO}_{7}$, Phys. Rev. B 88, 054422 (2013).

[23] M. Thede, T. Haku, T. Masuda, C. Baines, E. Pomjakushina, G. Dhalenne, A. Revcolevschi, E. Morenzoni, and A. Zheludev, Inhomogeneous ordering in weakly coupled Heisenberg $S=1 / 2$ chains with random bonds, Phys. Rev. B 90, 144407 (2014). 
[24] J. C. Bonner and M. E. Fisher, Linear magnetic chains with anisotropic coupling, Phys. Rev. 135, A640 (1964).

[25] D. C. Johnston, R. K. Kremer, M. Troyer, X. Wang, A. Klümper, S. L. Bud'ko, A. F. Panchula, and P. C. Canfield, Thermodynamics of spin $S=1 / 2$ antiferromagnetic uniform and alternating-exchange Heisenberg chains, Phys. Rev. B 61, 9558 (2000).

[26] M. Horvatić and C. Berthier, NMR studies of low-dimensional quantum antiferromagnets, in High Magnetic Fields, edited by C. Berthier, L. P. Lévy, and G. Martinez, Lecture Notes in Physics (Springer-Verlag, Berlin, 2002), Vol. 595, Chap. 7, pp. 191-210.

[27] F. Casola, T. Shiroka, V. Glazkov, A. Feiguin, G. Dhalenne, A. Revcolevschi, A. Zheludev, H.-R. Ott, and J. Mesot, Dimensional crossover of spin chains in a transverse staggered field: An NMR study, Phys. Rev. B 86, 165111 (2012).

[28] L. J. Azevedo, A. Narath, P. M. Richards, and Z. G. Soos, Highfield spin dynamics of the one-dimensional spin-1/2 Heisenberg antiferromagnet $\alpha$-bis ( $\mathrm{N}$-methylsalicylaldiminato copper) (II) ( $\alpha$-CuNSal), Phys. Rev. B 21, 2871 (1980).

[29] T. Moriya, Nuclear magnetic relaxation in antiferromagnetics, Prog. Theor. Phys. 16, 23 (1956).

[30] J. Herbrych, J. Kokalj, and P. Prelovšek, Local Spin Relaxation Within the Random Heisenberg Chain, Phys. Rev. Lett. 111, 147203 (2013).

[31] T. Moriya, Nuclear magnetic relaxation near the Curie temperature, Prog. Theor. Phys. 28, 371 (1962).

[32] F. Borsa, Phase transitions and critical phenomena in solids, in eMagRes, edited by R. K. Harris and R. E. Wasylishen (John Wiley \& Sons, Hoboken, NJ, 2007).
[33] M. Arai, M. Fujita, M. Motokawa, J. Akimitsu, and S. M. Bennington, Quantum Spin Excitations in the SpinPeierls System $\mathrm{CuGeO}_{3}$, Phys. Rev. Lett. 77, 3649 (1996).

[34] A. Zheludev, Interacting quantum spin chains, Appl. Phys. A 74, s1 (2002).

[35] I. A. Zaliznyak, H. Woo, T. G. Perring, C. L. Broholm, C. D. Frost, and H. Takagi, Spinons in the Strongly Correlated Copper Oxide Chains in $\mathrm{SrCuO}_{2}$, Phys. Rev. Lett. 93, 087202 (2004).

[36] B. Lake, D. A. Tennant, C. D. Frost, and S. E. Nagler, Quantum criticality and universal scaling of a quantum antiferromagnet, Nat. Mater. 4, 329 (2005).

[37] S. Sachdev, NMR relaxation in half-integer antiferromagnetic spin chains, Phys. Rev. B 50, 13006 (1994).

[38] R. Chitra and T. Giamarchi, Critical properties of gapped spinchains and ladders in a magnetic field, Phys. Rev. B 55, 5816 (1997).

[39] J. Sirker, R. G. Pereira, and I. Affleck, Diffusion and Ballistic Transport in One-Dimensional Quantum Systems, Phys. Rev. Lett. 103, 216602 (2009).

[40] H. Kühne, H.-H. Klauss, S. Grossjohann, W. Brenig, F. J. Litterst, A. P. Reyes, P. L. Kuhns, M. M. Turnbull, and C. P. Landee, Quantum critical dynamics of an $S=1 / 2$ antiferromagnetic Heisenberg chain studied by ${ }^{13} \mathrm{C}$ NMR spectroscopy, Phys. Rev. B 80, 045110 (2009).

[41] K. R. Thurber, A. W. Hunt, T. Imai, and F. C. Chou, ${ }^{17}$ O NMR Study of $q=0$ Spin Excitations in a Nearly Ideal $S=1 / 21 \mathrm{D}$ Heisenberg Antiferromagnet, $\mathrm{Sr}_{2} \mathrm{CuO}_{3}$, up to $800 \mathrm{~K}$, Phys. Rev. Lett. 87, 247202 (2001). 